\title{
Catalogue des types de poissons, amphibiens et reptiles du Muséum d'Histoire naturelle de Genève
}

par

\author{
Volker MAHNERT
}

Jusqu'à une époque récente, les vertébrés inférieurs n'avaient pas fait l'objet d'études systématiques au Muséum d'Histoire naturelle de Genève. Dans la seconde moitié du $\mathrm{XIX}^{\mathrm{e}}$ siècle et au début de celui-ci, seuls quelques types étaient déposés dans les collections. Il s'agissait des poissons décrits par Lunel (premier directeur du Muséum) et par Regan, ainsi que des reptiles décrits par Jan et Peracca.

Ce n'est que depuis une vingtaine d'années que l'étude des vertébrés inférieurs a pris une certaine importance dans notre institut. L'activité scientifique des chercheurs du Muséum et de nombreux échanges nous ont valu de recevoir du matériel type d'auteurs contemporains.

Ce catalogue contient tous les types (holotypes et autres catégories) (poissons: 26 espèces; amphibiens: 72 espèces et sous-espèces; reptiles: 45 espèces et sous-espèces). Chaque espèce est citée ainsi:

- nom donné dans la description originale

- date de la publication

- référence bibliographique

- catégorie de type et numéro de collection (conservation en alcool, sauf indication contraire)

— localité typique avec tous les détails disponibles.

A titre d'information et sous toute réserve, le nom actuel est donné, selon les ouvrages de révision récents. La bibliographie à la fin du catalogue ne répète pas les références des descriptions originales, mais renferme seulement les titres de publications concernant le statut des espèces citées.

Je tiens à remercier mon collègue Jean-Luc Perret ainsi que tous ceux qui m'ont fourni des informations sur les espèces citées ci-dessous ou qui ont réexaminé des types pour en éclaircir le statut actuel. Ils sont trop nombreux pour être énumérés ici. 


\section{PISCES}

\section{CHONDRICHTHYES}

\section{Pteroplatea binotata Lunel}

1879 Mém. Soc. Phys. Hist. nat. Genève 26 (2): 423-426, fig. 1a, b; 2.

Holotype: MHNG 1213.89.

1 ex., Brésil, Rio de Janeiro; $\mathrm{D}^{\mathrm{r}}$ Nägeli.

= Gymnura altavela (Linné)

\section{Osteichthyes}

Amphilius platychir cubangoensis Pellegrin

1936 Archos. Mus. Bocage 7: 56-57.

Paratype: MHNG 858.86.

1 ex., Angola, Cubango (Kuwangu) près de Vila da Ponte; A. Monard, 1928/29 (?).

\section{Aphyosemion cyanostictum Lambert et Géry}

1967 Biol. Gabon. 3: 308-310, fig. 4, pl. III.

Paratypes: MHNG 1221.18-23.

6 ex., Gabon, marigot affluent de la Nyabariéle après Ybiegn, PK 18-19 de la route Makakou-Libreville, bassin de l'Ivindo; G. Géry, 9.X.1964.

\section{Aphyosemion georgiae Lambert et Géry}

1968 Biol. Gabon. 3: 306-308, fig. 3, pl. III.

Paratypes: MHNG 1221.12-14.

3 ex., Gabon, marigot avant Belniga, bassin de l'Ivindo; J. et G. Géry, 14.IX.1964.

\section{Balistes mauritianus Regan}

1903 Revue suisse Zool. 11: 414-415, pl. 13, fig. 4.

Holotype: MHNG 665.43.

1 ex., Ile Maurice; H. de Saussure.

Regan a noté comme provenance de l'exemplaire: "Ile Maurice », sur l'étiquette se trouve l'origine: "Ile Bourbon = Réunion ».

\section{Bedotia madagascariensis Regan}

1903 Revue suisse Zool. 11: 416-417, pl. 14, fig. 2.

Holotype: MHNG 665.7.

1 ex., Madagascar, eau douce.

Un deuxième exemplaire (inscrit sous le numéro 1213.08) de même origine se trouvait dans le bocal avec le type: Pellegrin avait examiné les deux spécimens en 1935 et désigné l'exemplaire ayant servi à la description originale (in litt.). 


\section{Botia multifasciata Regan}

1905 Revue suisse Zool. 13: 389, pl. 5, fig. 3.

Holotype: MHNG 677.98.

1 ex., Chine, Tung-Kung, est de Canton, sur la rivière de l'Est; Kühne, 1903 (?).

FANG (1903) considère cette espèce comme synonyme de Botia (Hymenophysa) xanthi (Günther). D'après Monsieur $\mathrm{D}^{\mathrm{r}}$ Petru Banarescu (in litt.) l'espèce est conspécifique avec Leptobotia fasciata (Dabry) et représente une sous-espèce de celle-ci, Leptobotia fasciata multifasciata (Regan).

\section{Brama saussurei Lunel}

1866 Mém. Soc. Phys. Hist. nat. Genève 18: 185-191, pl. II, fig. 1-1i.

Holotype: MHNG 940.85.

1 ex., Cuba; H. de Saussure.

= Pseudotaractes saussurei (Lunel)

Bryconops (Creatochanes) inpai Knoeppel, Junk et Géry

1968 Amazoniana, Kiel 1 (3): 231-245, fig. 1b, 2, 3, 4.

Paratypes: MHNG 1336.55-75.

21 ex., Brésil, Lower Rio Negro region, Igarapé Barro Branco, a brook in the "Reserva Ducke» (INPA), about $30 \mathrm{~km}$ on the road from Manaus to Itacoatiara; E.-F. Fittkau, XI.1965.

\section{Doryichthys multiannulatus Regan}

1903 Revue suisse Zool. 11: 413-414, pl. 13, fig. 3.

Holotype: MHNG 665.51.

1 ex., Ile Maurice; $H$. de Saussure.

= Dunckerocampus multiannulatus (Regan)

Eleotris pectoralis Regan .

1903 Revue suisse Zool. 11: 415-416, pl. 13, fig. 2.

Holotype: MHNG 665.6.

1 ex., Madagascar, eau douce.

$=$ Eleotris tohizonae Steindachner

\section{Grasseichthys gabonensis Géry}

1964 C.r. hébd. Séanc. Acad. Sci. Paris 259: 4805-4807, fig.

Paratypes: MHNG 1221.15-17.

3 ex., Gabon, environs de Makokou, bassin de l'Ivindo, PK 25 de la route du Bouénli; J. Géry et J. Lambert, 19.X.1964.

\section{Gymnomuraena brevicauda Regan}

1903 Revue suisse Zool. 11: 414, pl. 13, fig. 1.

Holotype: MHNG 665.54.

1 ex., Ile Maurice; H. de Saussure.

$=$ Uropterygius bennetti Günther 


\section{Heros octofasciatus Regan}

1903 Revue suisse Zool. 11: 417-418, pl. 13, fig. 1.

Syntypes: MHNG 665.55.

9 ex., Mexique, Casamoloapan (Côte Orientale du Mexique); H. de Saussure, eau douce.

La description ne mentionne qu'un exemplaire; dans le bocal avec l'étiquette « dét. par C. T. Regan » se trouve la série de neuf exemplaires.

= Cichlasoma octofasciatum (Regan)

\section{Macrones argentivittatus Regan}

1905 Revue suisse Zool. 13: 390, pl. 5, fig. 2.

Lectotype: MHNG 677.99 (désigné par JAYARAM 1974).

1 ex., Chine, Cungkun, Canton, sur la rivière de l'est; Miss. Kühne, IX.1903.

Paralectotypes: MHNG 677.99b, c.

2 ex., même provenance.

$=$ Mystus argentivittatus (Regan)

\section{Marcusenius cubangoensis Pellegrin}

1936 Archos. Mus. Bocage 7: 48.

Paratype: MHNG 858.85.

1 ex., Angola, Cubango (Kuwangu); A. Monard 1932/33 (?).

\section{Muraena grandimaculis Regan}

1903 Revue suisse Zool.: 418, pl. 14, fig. 3.

Holotype: MHNG 665.39.

1 ex., Brésil, Bahia.

\section{Novacula temporalis Regan}

1905 Revue suisse Zool. 13: 392-393, pl. 6, fig. 3.

Holotype: MHNG 678.2.

1 ex., Ile Bourbon («Réunion »); H. de Saussure.

$=$ Hemipteronotus pentadactyla Linné

\section{Penetropteryx taeniocephalus Lunel}

1881 Mém. Soc. Phys. Hist. nat. Genève 27 (2): 275-278, fig. 1-1e.

Syntypes: MHNG 843.48.

3 ex., Ile Maurice.

Un des quatre syntypes a été échangé avec le Muséum de Gênes.

\section{Percichthys altispinis Regan}

1905 Revue suisse Zool. 13: 390-391, pl. 5, fig. 1.

Syntypes: MHNG 677.100.

2 ex., Argentine, Rio Colorado, Buenos Aires, eau douce.

La description était basée sur 3 exemplaires; deux seulement sont conservés dans la collection. 


\section{Phreatobius cisternarum Goeldi}

1904 C.R. 6e Congr. internat. Zool. Berne: 549.

Syntype (?): MHNG 1213.97.

1 ex., Brésil, Ile de Marajo (à l'embouchure de l'Amazone), au nord de Source, dans une citerne; E. Goeldi, 1903 (ou V. Ch. Miranda, 1905 ?).

MHNG 1505.91: 65 préparations microscopiques.

Deux exemplaires ont servi à la description, quatre autres furent trouvés deux ans plus tard. De ces six spécimens un est conservé en alcool dans notre collection, un deuxième dans les collections du Field Museum of Natural History, Chicago, sous le numéro du Carnegie Museum 7603. D'après ReICHEL (1927), trois de ces cinq exemplaires restés en Europe ont été montés en préparations microscopiques, un conservé en alcool et un dans la tétraline. Ce dernier est introuvable.

\section{Pseudochalceus longianalis Géry}

1972 Revue suisse Zool. 79 (2): 933-942, fig. 1-4.

Holotype: MHNG 1226.90.

1 ex., Sud de la Colombie, près de la frontière avec l'Equateur, versant pacifique: rio Guiza, affluent du rio Mira, à la Guayacana, Province Narino, alt. 240 m., coord. $1^{\circ} 27^{\prime} \mathrm{N}$ et $78^{\circ} 27^{\prime} \mathrm{O}$; K. von Sneidern, III.1958.

Paratypes: MHNG 1226.91-99.

9 ex., même localité et même date.

\section{Saccoderma robusta Dahl}

1955 Revista Linneana 1: 11-19 (publication introuvable).

Paratypes: MHNG 1066.39-42.

4 ex., Colombie, Rio Verde, Sinu.

\section{Sciaena (Bairdiella) bedoti Regan}

1905 Revue suisse Zool. 13: 391-392, pl. 6, fig. 1.

Syntype: MHNG 678.1.

1 ex., Cuba; Voyage H. de Saussure.

La description de l'espèce était basée sur deux exemplaires, il n'en reste qu'un exemplaire dans les collections.

= Bairdiella rochus (Cuvier)

\section{Solea borbonica Regan}

1905 Revue suisse Zool. 13: 393, pl. 6, fig. 2.

Holotype: MHNG 678.3.

1 ex., Ile Bourbon (= Réunion); H. de Saussure.

= Aesopia borbonica (Regan)

\section{Trichomycterus chaberti Durand}

1968 Annls. Spéléol. 23 (2): 344-352, fig. 2-4.

Paratypes: MHNG 1513.19-22.

Bolivie, grotte Umayalanta, à $8 \mathrm{~km}$ au N.W. du village de Torotoro (département de Potosi, province de Charcas) à $130 \mathrm{~km}$ au Sud de Cochabamba; J. Chabert, J. Meunier et H. Saavedra, 7.IX.1966. 


\section{AMPHIBIA}

\section{URODELA}

\section{Hydromantes italicus bonzanoi Bruno et Bologna}

1973 Atti Soc. ital. Sci. nat. Museo civ. Stor. nat. Milano 114 (1): 82-89, fig. 1. Paratype: MHNG 1520.12.

$1 \hat{o}^{\wedge}$, Italie, Ligurie, grotta detta «Tana Ia du Casà » $(537 \mathrm{Li}) 4^{\circ} 30^{\prime} 27^{\prime \prime}$ e $44^{\circ} 00^{\prime} 12^{\prime \prime}$; I.G.M.: F 91, Q II, S.E., Pieve di Teco. Situata sul versante Sud del Colle San Bartolomeo nell'alta Valle del torrente Impero, com. Caravonica, prov. Imperia, alt. $712 \mathrm{~m}$; C. Bonzano, 10.X.1971.

Paratype: MHNG 1520.13

1 ㅇ, Italie, Ligurie, " Tana de’ Fasceo », com. Molini di Prelà, nella valle del torrente Prino, in frazione Canneto; M. Bologna, 16.VII.1972.

\section{Hydromantes italicus strinatii Aellen}

1958 Senckenberg. biol. 39: 156, Abb. 1.

Holotype: MHNG 926.16.

1 ô, France, grotte d'Aspremont, Alpes-Maritimes; Ch. Roth, P. Strinati, V. Aellen, 13.X.1957.

Paratypes: MHNG 935.13.

1 \&, même provenance.

Paratypes: MHNG 903.21.

1 q, même localité; Korsakoff, 4.VIII.1938.

\section{ANURA}

Afrixalus congicus paradorsalis Perret

1960 Revue Zool. Bot. afr. 61: 370, pl. 2, fig. 1-8; pl. 3, fig. 1.

Holotype: MHNG 991.50.

1 ô., Cameroun, Foulassi, Sangmelima; J.-L. Perret, 1959.

Paratypes: MHNG 991.51-68 (991.65-68 donnés au Mus. Copenhague).

18 ô, Cameroun, Foulassi, Sangmelima, J.-L. Perret, 1959-1960.

Paratypes: MHNG 991.87-88.

2 ô, même provenance.

Paratypes: MHNG 991.69-86 (991.85-86 donnés au Mus. Copenhague).

16 + , même provenance.

Paratypes: MHNG 991.89-90.

2 \% , même provenance.

Paratypes: MHNG 991.91-99.

9 juv., même provenance.

Paratypes: MHNG 991.100.

Larves, même provenance.

$=$ Afrixalus paradorsalis Perret 
Afrixalus dorsalis laciniosus Perret

1960 Revue Zool. Bot. afr. 61: 369-370, pl. 1, fig. 1-5.

Holotype: MHNG 1035.39.

1 ô, Cameroun, Douala, J.-L. Perret, I.1959.

Paratypes: MHNG 1035.40-50.

11 ô, même provenance.

Paratype: MHNG 1035.51.

1 , même provenance.

Afrixalus lacteus Perret

1976 Bull. Soc. Neuchâtel. Sci. nat. 99: 25-27, pl. II.

Holotype: MHNG 1506.58.

1 ธิ, Cameroun, Nsoung, Mont Manengouba; J.-L. Perret, 29.III.1973.

Paratypes: MHNG 1506.59-96.

37 ô, 1 ㅇ, même provenance.

Paratypes: MHNG 1506.97.

1 \&, Cameroun, Foto, Bamiléké, alt. 1400 m., J.-L. Perret, XI.1972.

Afrixalus leucostictus Laurent

1950 Revue Zool. Bot. afr. 44: 7.

Paratypes: MHNG 1347.17-19.

3 ㅇ, Zaïre, Itula, 650 m., Terr. de Shabunda, Kivu; R. F. Laurent, 14.-19.V.1950.

Paratypes: MHNG 1347.20-21.

2 ô, Zaïre, Katshungu, 700 m., Terr. de Shabunda, Kivu; R. F. Laurent, 28.30.I.1950.

Paratypes: MHNG 1347.22-24.

3 ô, Zaïre, Kanituga, 1050 m., Terr. de Mwenga, Kivu; R. F. Laurent, 4.-9.V.1950.

Paratypes: MHNG 1347.25-29.

5 ô, Zaïre, Makese, 650 m., est de Shabunda, nord de l'Ulindi, Kivu; R. F. Laurent, 31.I.1950.

Arthroleptis adelphus Perret

1966 Zool. Jb. Syst. 93: 397-399, fig. 47.

Holotype: MHNG 1042.12.

1 i, Cameroun, Foulassi, Sangmelima; J.-L. Perret, 1961.

Paratypes: MHNG 1042.13-19.

7 +, Cameroun, Foulassi; J.-L. Perret, 1960-1961.

Paratypes: MHNG 1042.20-24.

5 q, même provenance.

Paratypes: MHNG 1042.25-28.

4 juv., même provenance.

Arthroleptis pyrrhoscelis Laurent

1952 Revue Zool. Bot. afr. 46: 28-30.

Paratypes: MHNG 1055.7-9.

3 ex., Zaïre, Haute Lubitshakom, 1900-2000 m., Terr. de Fizi, Kivu; R. F. Laurent, 26.-30.X.1950.

= Schoutedenella pyrrhoscelis (Laurent) 
Arthroleptis troglodytes Poynton

1963 Annls. Natal Mus. 15: 327.

Paratypes: MHNG 1511.74-75.

1 ô, 1 \&, Rhodésie, Western Range, Chimanimani Mountains; D. G. Broadley, (Umtali Museum 3732).

\section{Bufo bambutensis Amiet}

1972 Annls. Fac. Sci. Cameroun 11: 132-135, fig. 1b, pl. 1: c.

Holotype: MHNG 1253.92.

1 ex., Cameroun, Mts Bamboutos, env. 2600 m.; J.-L. Amiet, 21.II.1971 (nº 71.026).

$=$ Werneria bambutensis (Amiet)

Bufo funereus upembae Schmidt et Inger

1959 Expl. Parc Nat. Upemba, fasc. 56: 17.

Paratypes: MHNG 1020.82-83.

2 ô, Zaïre, Upemba, Gorges de la Pelenge, affl. Lufira; Miss. G. F. de Witte, VI.1947.

Paratypes: MHNG 1020.84-87.

4 , , même provenance.

= Bufo fuliginatus Witte (Perret, comm. pers.)

\section{Bufo mertensi Amiet}

1972 Annls. Fac. Sci. Cameroun 11: 125-129, fig. 1b, pl. 1: b, pl. 2: c, d.

Holotype: MHNG 1253.91.

1 ex., Cameroun, Mt. Nlonako, env. 1000 m.; J.-L. Amiet 23.I.1970 (no 70.380).

$=$ Werneria mertensiana (Amiet)

Bufo pentoni tihamicus Balletto et Cherchi

1973 Boll. Mus. Ist. Biol. Univ. Genova 41: 114-118, fig. 5; pl. 2, fig. 2-4.

Paratypes: MHNG 1185.79-81.

3 ô, Yemen, El Bazra; G. Scortecci, VIII.1965.

Paratypes: MHNG 1185.82-84.

3 , même provenance.

\section{Bufo perreti Schiøtz}

1963 Vidensk. Medd. fra Dansk naturh. Foren 125: 17-22, fig. 1-4; pl. 2, fig. 1-4.

Paratype: MHNG 1054.10.

1 ô, Nigéria, Idanre Hills, près Idanre Resthouse, prov. Ondo; A. Schiøız, 4.VII.1961 (Mus. Copenhague $n^{\circ}$ R 13530).

\section{Bufo scorteccii Balletto et Cherchi}

1970 Boll. Mus. Ist. biol. Univ. Genova 38, n 260: 34-38, fig. 5B, 6B.

Paratype: MHNG 1240.32.

1 ô, Yemen, Mafhag, Oued Kalila; G. Scortecci, 28.VIII.1965.

Paratype: MHNG 1240.31.

1 ㅇ, même localité. 


\section{Bufo tandyi Amiet}

1972 Annls. Fac. Sci. Cameroun, no 11: 129-132, fig. 1, pl. 1:a, pl. 2: a, b.

Holotype: MHNG 1253.93.

1 ex., Cameroun, Nsoung, env. 1400 m., J.-L. Amiet, 29.III.1971 (nº 71.912).

$=$ Werneria tandyi (Amiet)

\section{Callixalus pictus Laurent}

1950 Revue Zool. Bot. afr. 44 (1): 5-7.

Paratype: MHNG 1197.17.

1 ๙ิ, Zaïre, Haute Luvubu, 2550-2600 m., Terr. d’Uvira, Kivu; R. F. Laurent, 28.-30.IX.1950.

Paratype: MHNG 1197.18.

1 \&, même provenance.

\section{Cardioglossa gratiosa Amiet}

1972 Biol. Gabon. 8 (2): 221-227, fig. 10-11, pl. 3 : fig. 1.

Holotype: MHNG 1253.85.

1 ô, Cameroun, Ongot, env. 750 m., bord du Nga; J.-L. Amiet, 15.I.1971 (nº 71.070).

\section{Cardioglossa melanogaster Amiet}

1972 Biol. Gabon. 8 (2): 212-217, fig. 4, 5b, 6, pl. 2: fig. 1, 2.

Holotype: MHNG 1253.86.

1 ô, Cameroun, Mwakoumel, env. 1250 m.; J.-L. Amiet, 26.V.1971 (n ${ }^{\circ}$ 71.765).

\section{Cardioglossa oreas Amiet}

1972 Biol. Gabon. 8 (2): 217-221, fig. 7-8.

Holotype: MHNG 1253.87.

1 , Cameroun, Mts. Bamboutos, env. 2650 m.; J.-L. Amiet 27.I.1971 (no 71.123).

\section{Cardioglossa trifasciata Amiet}

1972 Biol. Gabon. 8 (2): 204-209, fig. 1, pl. 1 : fig. 1.

Holotype: MHNG 1253.88.

1 ô, Cameroun, Nsoung, 1750-1800 m.; J.-L. Amiet 2.XII.1970 (nº 70.599).

\section{Cardioglossa venusta Amiet}

1972 Biol. Gabon. 8 (2): 209-212, fig. 2, pl. 1 : fig. 2.

Holotype: MHNG 1253.89.

1 ô, Cameroun, Fotabong, 950-1000 m.; J.-L. Amiet 25.V.1971 (n $\left.{ }^{\circ} 71.872\right)$.

\section{Chrysobatrachus cupreonitens Laurent}

1951 Revue Zool. Bot. afr. 44 (4): 376-381.

Paratypes: MHNG 1054.83-94.

6 ô, 6 ․ Zaïre, Haute Luvubu, 2550 m., bassin de la Ruzizi, Terr. d’Uvira, Kivu;

R. F. Laurent, 23.-30.IX.1950. 
Hylarana acutirostris longipes Perret

1960 Bull. Soc. Neuchâtel. Sci. nat. 83: 97-99, fig. 4.

Holotype: MHNG 986.24.

1 今ิ, Cameroun, Bangwa; J.-L. Perret, 1959.

Paratypes: MHNG 986.25-26.

2 ô, même provenance.

$=$ Hylarana longipes Perret

Hymenochirus boettgeri camerunensis Perret et Mertens

1957 Bull. I.F.A.N. Sér. A, 19: 552-554, fig. 1.

Paratypes: MHNG 917.94-95.

2 , Cameroun, Foulassi; J.-L. Perret, 1954-1955.

\section{Hymenochirus boulengeri Witte}

1930 Revue Zool. Bot. afr. 19: 239-240.

Paratypes: MHNG 1018.37-42.

6 ex., Zaïre, Koteli, Uélé; H. Schouteden, I.1925 (éch. R. G. Mus. Congo, nº 42414246).

Hyperolius castaneus constellatus Laurent

1951 Revue Zool. Bot. afr. 44 (4): 369-371.

Paratypes: MHNG 1202.89-100.

12 ô, Zaïre, Haute Luvubu, 2550 m., bassin de la Ruzizi, Terr. d'Uvira, Kivu; R. F. Laurent, 23.-30.IX.1950 ( $\left.\mathrm{n}^{\circ} 1940\right)$.

Paratypes: MHNG 1203.1-3.

3 ồ, même provenance.

Paratypes: MHNG 1203.4-13.

10 \&, même provenance.

Hyperolius chrysogaster Laurent

1950 Revue Zool. Bot. afr. 44 (1): 13-14.

Paratypes: MHNG 1205.61-65.

5 ô, Zaïre, Kamituga, 1000-1100 m., Terr. Mwenga, Kivu; R. F. Laurent, 9.VIII.1950 (n 411).

Hyperolius frontalis Laurent

1950 Revue Zool. Bot. afr. 44 (1): 11-12.

Paratypes: MHNG 1204.97-100, 1205.1.

5 ô, Zaïre, Kamituga, 1000-1100 m., Terr. Mwenga, Kivu; R. F. Laurent, 4.10.V.1950 (n 1311).

Hyperolius karissimbiensis intermedius Laurent

1950 Revue Zool. Bot. afr. 44 (1): 11.

Paratypes: MHNG 1204.33-37.

5 ô, Urundi, Bitare, 1550 m., Terr. de Kitega; R. F. Laurent, 16.-19.II.1950 (nº 1920).

$=$ Hyperolius viridiflavus intermedius Laurent 
Hyperolius lateralis bogerti Laurent

1952 Revue Zool. Bot. afr. 46 (3-4): 277-278.

Paratypes: MHNG 1202.23-27.

5 o, Rwanda, Remera, Lac Luhondo, 1770 m., Terr. de Ruhengeri; R. F. Laurent, 23.-25.I.1951 ( $\left.n^{\circ} 1786\right)$.

Hyperolius lateralis bururiensis Laurent

1950 Revue Zool. Bot. afr. 44 (1): 8-9.

Paratypes: MHNG 1206.19-23.

5 ô, Urundi, Bururi, 1850-1950 m.; R. F. Laurent, 21.-27.XII.1949 (n ${ }^{\circ}$ 1783).

Hyperolius lateralis capnodogaster Laurent

1951 Revue Zool. Bot. afr. 44 (4): 374-375.

Paratypes: MHNG 1202.64-68.

5 ô, Urundi, marais de la Tshizanie, Kinazi, $1350 \mathrm{~m}$., Terr. de Muhinga; R. F. Laurent, 17.-18.IV.1950 ( $n^{\circ}$ 97).

Hyperolius lateralis guibei Laurent

1951 Revue Zool. Bot. afr. 44 (4): 373-374.

Paratypes: MHNG 1203.14-18.

5 ô, Urundi, marais Rugushu, Kayanza, 1900 m., Terr. de Ngozi; R. F. Laurent, 20.-21.II.1950 ( $\mathrm{n}^{\circ}$ 1764).

Hyperolius lateralis kochi Laurent

1951 Revue Zool. Bot. afr. 44 (4): 375.

Paratypes: MHNG 1207.43-47.

5 ô, Zaïre, Itula, 650 m., Terr. de Shabunda, Kivu; R. F. Laurent. 14.-19.V.1950 (n $\left.\mathrm{n}^{\circ} 76\right)$.

Hyperolius lateralis pulcherrimus Laurent

1952 Revue Zool. Bot. afr. 46 (3-4): 278-279.

Paratypes: MHNG 1204.43-47.

5 ô, Rwanda, marais de la Rusumu, 2100 m., Terr. de Ruhengeri; R. F. Laurent, 28.-29.I.1951 ( $\left.\mathrm{n}^{\circ} 1724\right)$.

Hyperolius lateralis subolivaceus Laurent

1952 Revue Zool. Bot. afr. 46 (3-4): 277.

Paratypes: MHNG 1202.28-32.

5 ô, Zaïre, marais de Kasha, Lemera, alt. 1550 m., Terr. d’Uvira, Kivu; R. F. Laurent, 15.-17.II.1951 ( $\left.\mathrm{n}^{\circ} 1633\right)$.

Hyperolius leucotaenius allogynus Laurent

1952 Revue Zool. Bot. afr. 46 (1-2): 34.

Paratypes: MHNG 1204.92-96.

5 ô, Zaïre, Haute Lubitshako, 1900-2000 m., Terr. de Fizi, Kivu; R. F. Laurent, 26.-30.X.1950 ( $\left.{ }^{\circ} 1631\right)$. 


\section{Hyperolius leucotaenius Laurent}

1950 Revue Zool. Bot. afr. 44 (1): 14-15.

Paratypes: MHNG 1204.87-91.

5 đૈ, Zaïre, Lviko, 1900 m., Terr. Fizi, Sud-Kivu; R. F. Laurent, 29.-30.VI.1950 (n $\mathrm{n}^{\circ}$ 72).

\section{Hyperolius mosaicus Perret}

1959 Revue suisse Zool. 66: 716-721, fig. 5, 6.

Holotype: MHNG 965.12.

1 ô, Cameroun, Ngam, Sangmelima, alt. 710 m., forêt; J. L. Perret, 10.III.1959.

Paratype: MHNG 965.13.

1 ㅇ, même provenance, X.1958-III.1959.

Paratypes: MHNG 965.14-21.

8 ô, même provenance.

En 1958, LAURent a rapporté à Hyperolius acutirostris Peters des exemplaires de mosaicus du British Museum ce qui a conduit Perret (1966) (en accord avec l'identification de Laurent) à désigner un néotype d'acutirostris en choisissant l'holotype de mosaicus. Or les deux espèces sont bien distinctes actuellement; le néotype d'acutirostris a été invalidé (PERRET 1975).

\section{Hyperolius nasutus igbettensis Schiøtz}

1963 Vidensk Medd. fra Dansk. naturh. Foren. 125: 64, fig. 22, pl. 1: fig. 13-14, pl. 4: fig. 10.

Paratype: MHNG 1039.12.

1 ㅇ, Nigeria Ouest, Igbetti Village, Oyo, 25.X.1958 (Mus. Copenhague nº RO 71817).

Paratypes: MHNG 1039.13-15.

3 ડ̂, même provenance (Mus. Copenhague nº RO 71827, 71828, 71829).

Hyperolius nitidulus aureus Perret

1966 Zool. Jb. Syst. 93: 416-417, fig. 57 A.

Holotype: MHNG 1038.39.

1 ô., Cameroun, Ngaoundéré; J.-L. Perret, 1960.

Paratypes: MHNG 1038.40-41.

2 , même provenance.

Paratypes: MHNG 1038.42-100.

59 ô, même provenance.

$=$ Hyperolius viridiflavus nitidulus Peters

\section{Hyperolius nitidulus bangwae Perret}

1966 Zool. Jb. Syst. 93: 415-416, fig. 57 B.

Holotype: MHNG 1039.2.

1 ơ, Cameroun, Bangwa; J.-L. Perret, 1959.

Paratypes: MHNG 1039.3-11.

9 ô, même provenance.

Paratypes: MHNG 960.63-88.

26 ơ, même provenance; J.-L. Perret, 1957.

$=$ Hyperolius viridiflavus bangwae Perret 


\section{Hyperolius obstetricans Ahl}

1931 Mitt. zool. Mus. Berlin 17: 90.

Néotype: MHNG 995.48 (désigné par Perret 1966: 409-410, fig. 54).

1 ô, Cameroun, Foulassi, rivière Lobo; J.-L. Perret, 1961.

\section{Hyperolius platyceps olbrechtsi Laurent}

1952 Revue Zool. Bot. afr. 46 (3-4): 275-276.

Paratypes: MHNG 1204.48-57.

10 oิ, Tanzanie, Nyunzu, alt. 700 m., Terr. d'Albertville; R. F. Laurent, 11.14.XII.1950 ( $\left.\mathrm{n}^{\circ} 1885\right)$.

\section{Hyperolius viridiflavus hybridus Laurent}

1951 Revue Zool. Bot. afr. 44 (4): 375-376.

Paratypes: MHNG 1204.82-86.

5 ô, Rwanda, Etang de la Mukura, 1700 m., près d'Astrida; R. F. Laurent, 15.IV.1950 (n० 74).

Kassina senegalensis ruandae Laurent

1956 Folia Sci. Afr. Centr. II, nº 2: 17.

Paratypes: MHNG 1205.53-60.

7 ô, 1 ㅇ, Rwanda, Butare; R. F. Laurent, 24.XI.1950 (nº 64d).

\section{Leptodactylodon mertensi Perret}

1959 Bull. Soc. Neuchâtel. Sci. nat. 82: 247-249, pl. VII, fig. 1.

Holotype: MHNG 951.39.

1 ô, Cameroun, Nsoung près de Nkongsamba, Mt. Manengouba, $1400 \mathrm{~m}$., forêt de montagne; J.-L. Perret, 1957.

Leptopelis anchietae nordequatorialis Perret

1966 Zool. Jb. Syst. 93 : 434-435, fig. 87.

Holotype: MHNG 1004.8.

1 ô, Cameroun, Bangwa; J.-L. Perret, 1959.

Paratypes: MHNG 1004.9-18.

10 ô, même provenance; (6 ex. échangés).

Paratypes: MHNG 953.15-23.

9 ô, même provenance; J.-L. Parret, 1957 (1 ex. échangé).

Paratype: MHNG 953.24.

1 ô, Cameroun, Foumban; J.-L. Perret, 1957.

$=$ Leptopelis nordequatorialis Perret

Leptopelis modestus fiziensis Laurent

1973 Annls. Mus. roy. Afr. centr., Tervuren, ser. in-8, Sci. Zool. 202: 39-42, fig. 36. Paratypes: MHNG 1205.33-42.

9 juv., 1 ô, Zaïre, Mokanga, 1170 m., Terr. Fizi, Sud-Kivu; R. F. Laurent, 22.27.III.1956 ( $\left.\mathrm{n}^{\circ} 1933\right)$. 
Leptopelis ocellatus schiøtzi Laurent

1973 Annls. Mus. roy. Afr. centr., Tervuren, ser. in-8, Sci. Zool. 202: 26-28, fig. 28. Paratypes: MHNG 1202.84-88.

5 ô, Zaïre, Omaniundu, Terr. Lodj, Sankuru.

\section{Leptopelis rufus Reichenow}

1874 Arkiv Naturg. 40: 287-298.

Néotype: MHNG 1324.65 (désigné par Perret 1973: 88, fig. 1, 2).

1 ô, Cameroun, Nkongock; J.-L. Perret, 27.III.1973.

\section{Megalixalus leptosomus upembae Laurent}

1941 Revue Zool. Bot. afr. 35 (1): 125-127, fig. 3.

Paratypes: MHNG 1197.9-12.

4 ․ Zaïre, Nyonga (Ht. Luapula), Katanga; Bayet, 1926 (R. G. Mus. Congo 5480-83).

= Afrixalus upembae (Laurent)

\section{Nectophrynoides cryptus Perret}

1971 Annls. Fac. Sci. Cameroun, no 6: 104-107, fig. 1, 7, 8.

Paratypes: MHNG 1221.62-63.

2 ô, Tanzanie, Nyingwa, Mts. Uluguru, 2200 m., A. Loveridge, 28.X.1926.

Paratypes: MHNG 1221.64-67.

4 , même provenance.

Paratypes: MHNG 1240.30-31.

1 ऽิ, 1 q, Tanzanie, Vituri, Mts. Uluguru; A. Loveridge, 30.X.1926 (Mus. Comp. Zool. 12.520, 14.496).

Paratypes: MHNG 1352.44-46.

2 ㅇ, 1 §ิ, Tanzanie, Mts Uluguru; W. Colley (National Mus. Nairobi, A 310/2, 3, 6).

\section{Nectophrynoides minutus Perret}

1972 Annls. Fac. Sci. Univ. Cameroun no 11: 106-110, fig. 6, 8, 9.

Paratype: MHNG 1240.42.

1 q, Tanzanie, Bagilot, Mts. Uluguru, 2200 m.; A. Loveridge, X.1926.

Paratype: MHNG 1240.43.

1 ô, même provenance.

\section{Nectophrynoides mirei Perret}

1971 Annls. Fac. Sci. Cameroun, no 6: 100-104, fig. 2, 6.

Holotype: MHNG 1182.77.

1 ô, Cameroun, Mont Okou, 2500 m.; P. B. de Miré, 15.II.1970.

Paratypes: MHNG 1182.78-79.

2 ㅇ, même provenance.

Paratypes: MHNG 1182.80-82.

3 ô, même provenance.

Paratypes: MHNG 1182.83-95.

13 juv., même provenance.

$=$ Wolterstorffina mirei (Perret) 


\section{Petropedetes perreti Amiet}

1973 Bull. I.F.A.N. 35, sér. A, nº 2: 463-468, fig. 1 b.

Holotype: MHNG 1253.90.

1 ex., Cameroun, Mt. Manengouba, Nsoung, 1400-1500 m.; J.-L. Amiet (n 71.196).

\section{Phrynobatrachus aelleni Loveridge}

1955 Revue suisse Zool. 62: 145-147, fig. 2.

Holotype: MHNG 1056.97.

1 ô, Côte d'Ivoire, Adiopodoumé/Abidjan; V. Aellen, 22.VI.1953 (n 538).

\section{$=$ Phrynobatrachus plicatus (Günther)}

Phrynobatrachus anotis Schmidt et Inger

1959 Expl. Parc Nat. Upemba, fasc. 56: 139-143, fig. 58, 59, pl. V, 4.

Paratypes: MHNG 1053.62-73.

12 ex., Zaire, Lusinga, affluent dr. Lufwu et affluent Lufira, 1810 m.; Miss G. F. de Witte (R. G. Mus. Congo 89528-89539), 23.III.-5.IV.1947.

\section{Phrynobatrachus cornutus annulatus Perret}

1966 Zool. Jb. Syst. 93: 365.

Holotype: MHNG 961.81.

1 ô, Guinée, Zouguépo; M. Lamotte, X.1956.

Paratype: MHNG 961.82 .

1 , même provenance.

= Phrynobatrachus annulatus Perret

Phrynobatrachus cricogaster Perret

1957 Revue suisse Zool. 64 (3): 527-530, fig. 1, 2.

Holotype: MHNG 923.31.

1 ô, Cameroun, Nsoung, Massif du Manengouba, $30 \mathrm{~km}$ de Nkongsamba, $1000 \mathrm{~m}$.; forêt de montagne; J.-L. Perret, 7.IX.1956.

Paratypes: MHNG 923.32-33.

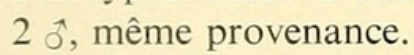

Phrynobatrachus cryptotis Schmidt et Inger

1959 Expl. Parc nat. Upemba, fasc. 56: 143-151, fig. 58, 60; pl. V, 5.

Paratypes: MHNG 981.98-100, 982.1-3.

6 ex., Zaïre, Mukana, marais près de Lusinga, 1810 m.; G. F. de Witte, 3.-4.I.1959.

Phrynobatrachus maculiventris Guibé et Lamotte

1958 Bull. Mus. natn. Hist. nat. Paris (2) 30: 255-257, fig. 1.

Paratypes: MHNG 961.96-97.

2 ex., Guinée, Mare Doromoux (forêt), Mt. Nimba; M. Lamotte, X.1956.

= Phrynobatrachus fraterculus (Chabanaud) 
Phrynobatrachus parogoensis Loveridge

1955 Revue suisse Zool. 62: 147-148.

Holotype: MHNG 1056.98.

1 q gravide, Côte d'Ivoire, Ndzida près de la côte; V. Aellen, 25.V.1953 (n ${ }^{n}$ 458).

= Phrynobatrachus accraensis $(\mathrm{Ahl})$

\section{Phrynobatrachus werneri hylaios Perret}

1959 Bull. Soc. Neuchâtel Sci. nat. 82: 251.

Holotype: MHNG 964.100.

1 ô, Cameroun, Foulassi, Sangmelima, 710 m.; forêt; J.-L. Perret, 1959.

Paratypes: MHNG 962.24-43.

20 ठิ , même provenance; J.-L. Perret, 1957.

Paratypes: MHNG 962.44-68.

25 , même provenance.

Paratype: MHNG 965.22.

1 + , même provenance; J.-L. Perret, 1959.

$=$ Phrynobatrachus hylaios Perret

\section{Pseudophryne viviparus Tornier}

1905 Sitzb. preuss. Akad. Wiss. Berlin 2: 855-857.

Néotype: MHNG 1221.55 (désigné par Perret 1972: 112-114).

1 oิ, Tanzania, Morogoro, Mts. Uluguru; P. Miles, X.1964.

$=$ Nectophrynoides vivipara (Tornier)

Ptychadena chrysogaster Laurent

1954 Annls. Mus. roy. Congo belge, Ser. 8, Sci. Zool., 34: 18-22, pl. I, fig. 3; pl. II, fig. 2 ; pl. III, fig. 5-6; pl. IV, fig. 3, 5.

Paratypes: MHNG 1054.50-55.

6 ex., Zaïre, Lulenga, Kivu; Dr. Schouteden, XI.-XII.1925 (R.G. Mus. Congo 3452-3457).

Rana (Ptychadena) obscura Schmidt et Inger

1959 Expl. Parc nat. Upemba, fasc. 56: 85-90; fig. 41-42.

Paratypes: MHNG 1954.15-21.

7 ex., Zaïre, gorges de la Pelenge, affl. de Lufira, 1250 m.; G. F. de Witte 21.V.-15.VI.1947 (R.G. Mus. Congo 68579-764).

$=$ Ptychadena obscura (Schmidt et Inger)

\section{Rana ruwenzorica Laurent}

1972 Expl. Parc nat. Virunga, 2e série, fasc. 22: 93-103, pl. I, fig. 4; pl. III, fig. 1-3; pl. IV, fig. 3; pl. X, fig. 2.

Paratypes: MHNG 1203.68-77.

5 ô, 5 †, Zaïre, Riv. Nyamwamba, 2000 m., Ruwenzori; coll. de Wilde, 17.IV.1952 $\left(n^{\circ} .1685\right)$. 
Rana (Ptychadena) upembae Schmidt et Inger

1959 Expl. Parc nat. Upemba, fasc. 56: 111.

Paratypes: MHNG 1054.11-14.

5 ex., Zaïre, Munoi, bifurcation, riv. Lupiala, affl. dr. Lufira, Katanga, 890 m.; G. F. de Witte, 29.V-11.VI.1948.

= Ptychadena upembae (Schmidt et Inger)

\section{Schoutedenella sylvatica Laurent}

1954 Annls. Mus. roy. Congo belge, Zool. 1: 38.

Paratypes: MHNG 1041.6-7.

2 ô, Zaïre, Buta; G. F. de Witte, II.1925. (R.G. Mus. Congo 8774-8785).

Paratypes: MHNG 1041.8-17.

10 \% , même provenance.

Xenopus laevis sudanensis Perret

1966 Zool. Jb. Syst. 93: 301-302, fig. 7.

Holotype: MHNG 1017.74.

1 ô, Cameroun, Ngaoundéré, Adamoua; J.-L. Perret, 1960.

Paratypes: MHNG 1017. 75-100.

26 ô, même provenance.

Paratypes: MHNG 1018.1-10.

10 . , même provenance.

Paratypes: MHNG 1018.11-25.

15 juv., même provenance.

Paratype: MHNG 1018.34.

1 +, Cameroun, Mbé, Duru; J.-L. Perret, 1960.

\section{REPTILIA}

SAURIA

Ablepharus duruarum Monard

1949 Revue suisse Zool. 56: 741-745, fig. 5.

Paratype: MHNG 1055.69.

1 ex., Cameroun, Mayo Zoro, Ngaouyanga (100 km au nord de Ngaoundéré, sur la Bénoué supérieure); A. Monard et V. Aellen, VII.-VIII.1947.

L'émendation: duruarus (GREeR 1974) n'est pas correcte.

= Afroablepharus duruarum (Monard)

\section{Chamaeleo eisentrauti Mertens}

1968 Bonn. zool. Beitr. 19 (1/2): 70-72, Abb. 1, 2.

Paratype: MHNG 1229.99.

1 ․, Cameroun, Dikume, Monts Rumpi, 1150 m.; M. Eisentraut, 17.II/5.III.1967. 


\section{Cnemaspis quattuorseriatus dilepis Perret}

1963 Revue suisse Zool. 70 (1): 48-49, fig. 1.

Holotype: MHNG 1005.69.

1 ô, Cameroun, Foulassi, Sangmelima, forêt; J.-L. Perret, 27.VIII. 1961.

\section{Eremias (Taenieremias) benuensis Monard}

1949 Revue suisse Zool. 56: 737-741, fig. 4.

Lectotype: MHNG 1055.62.

1 , Cameroun, Mayo Zoro, Ngaouyanga (100 km au nord de Ngaoundéré, sur la Bénoué supérieure); A. Monard et V. Aellen, 15.VII.1947, (coll. Monard $\left.\mathrm{n}^{\circ} .998\right)$.

\section{$=$ Eremias guineensis Boulenger}

\section{Lygodactylus angularis dysmicus Perret}

1963 Revue suisse Zool. 70: 53-55, fig. 3.

Holotype: MHNG 1005.72.

1 ô, Cameroun, Foulassi, Sud Cameroun forestier, à la limite nord-occidentale du bassin du Congo; J.-L. Perret, IX.1961.

$=$ Lygodactylus gutturalis dysmicus Perret

\section{Lygosoma rohdei Müller}

1910 Abh. bayer. Akad. Wiss. 2,24 (3): 585-587, fig. 3.

Néotype: MHNG 963.40 (désigné par FuHN 1972: 263-264, pl. II, fig. 7, pl. III, fig. 11, pl. VI, fig. 30).

1 ô, Cameroun, Ngam, Sangmelima; J.-L. Perret, 14.V.1957.

$=$ Panaspis rohdei (Müller)

\section{Mabuia striata angolensis Monard}

1937 Archos. Mus. Bocage 8: 89-90.

Paratype: MHNG 858.95.

1 ex., Angola, Kuvangu (ou Bimbi ?); A. Monard, 1932-1933.

\section{Millotisaurus mirabilis Pasteur}

1962 C.R. Soc. Sc. nat. Phys. Maroc 28: 65-66.

Paratype: MHNG 993.78.

1 ex., Madagascar, Mont Tsiafajavona, massif Ankaratra, 2300-2500 m.; J. Millot, 8.-9.IX.1959.

\section{Neusticurus tuberculatus Shreve}

1935 Occ. Pap. Boston Soc. nat. Hist. 8: 209.

Paratype: MHNG 1069.96.

1 ex., Equateur, Pastaza Riv., près de Canelos, Sarayacu.

$=$ Neusticurus ecpleopus Cope 


\section{Panaspis amieti Perret}

1973 Revue suisse Zool. 80 (2): 617-620, fig. 5, pl. VI.

Holotype: MHNG 1240.47.

1 今. Cameroun, Mont Kala, 800 m., $3^{\circ} 52^{\prime} \mathrm{N}, 11^{\circ} 31^{\prime} \mathrm{E}$, (Yaoundé); J.-L. Amiet, 1969.

Paratype: MHNG 1240.48 .

1 ô, même provenance.

Paratype: MHNG 1253.95.

1 ô juv., Cameroun, Boga, Eséka, 300 m.; J. L. Amiet, 6.I.1968.

\section{Panaspis fuhni Perret}

1973 Revue suisse Zool. 80 (2): 621-624, fig. 6, pl. II.

Holotype: MHNG 963.41.

1 , , Cameroun, Abong Mbang, $3^{\circ} 59^{\prime} \mathrm{N}, 13^{\circ} 11^{\prime} \mathrm{E}, 650$ m.; J.-L. Perret, 8.V.1957.

\section{Saurodactylus mauritanicus brosseti Bons et Pasteur}

1957 Bull. Soc. sc. nat. phys. Maroc 37 (3): 178-185, 192-195; fig. 1, 2, 3, 5; pl. IV. Paratype: MHNG 993.24.

1 ex., Maroc, basse vallée de l'oued Sousse; Smirnoff, été 1956.

\section{OPHIDIA}

\section{Atheris katangensis Witte}

1953 Explor. Parc nat. Upemba 6: 301-303, fig. 107, pl. III, fig. 4, pl. XII, fig. 2. Paratype: MHNG 1324.50.

1 , Zaïre, Mubale-Munte (région du confluent), sous-affluent et affluent de la rive droite de la Lufira, 1480 m.; Miss. G. F. de Witte, 7.V.1947 (coll. Kramer 9137).

Atractaspis coalescens Perret

1960 Revue suisse Zool. 67 (1): 130-131, fig. 1-3.

Holotype: MHNG 975.65.

1 †, Cameroun, Bangwa, en savane montagneuse du Bamiléké, 1400 m.; J.-L. Perret, 4.VII.1959.

Brachyophis revoili krameri Lanza

166 Monit. zool. Ital. 74 (1-3): 41-42, fig. 1, pl. 1, 5-7.

Paratype: MHNG 1324.13.

1 ô juv., Somalie septentrionale, Geriban, ca. $\left.48^{\circ} 40^{\prime} \mathrm{E} \times 7^{\circ} 15^{\prime} \mathrm{N}\right)$; III Missione Biologica in Somalia dell'Universita di Firenze (acquistato da un nativo), 16.VIII.1964 (coll. Kramer no. 5927).

Calamaria picteti Peracca

1899 Revue suisse Zool. 7: 325-326, pl. 14, fig. 1-3.

Holotype: MHNG 748.36.

1 ex., Borneo, Sarawak; Voyage M. Bedot-C. Pictet.

$=$ Calamaria bicolor Dum. \& Bibr. 


\section{Cemophora copei Jan}

1863 Arch. Zool. Anat. Fisiol. Modena 2 (2): 231.

Holotype: MHNG 524.74.

1 ex., Etats-Unis, Tennessee; H. de Saussure?

= Cemophora coccinea (Blumenb.)

\section{Coluber viridiflavus kratzeri Kramer}

1970 Lavori Soc. ital. Biogeografia, N.S. 1: 668-670, Taf. I, fig. 1-3, Taf. II, fig. 4. Paratypes: MHNG 1324.77-93.

Italie, Ile de Monte Cristo (Mer Tyrrhénienne); E. Kramer, J. Kretz, R. Jordi:

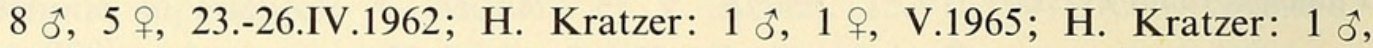
IV.1964; H. Kratzer: 1 ô, 1966; (Coll. Kramer nº. 3798, 3799, 3800, 3802, $3803,3804,3805,3806,3814,3815,3816,3817,3818,5548,5769,9279$, 9876).

Dryophis pulverulentus Jan

1863 Elenc. sist. ofidi : 88; et Icon. Gen. 32. pl. 5, fig. 1; 1869.

Holotype: MHNG 524.1 = 1198.71 .

1 ex., Ceylan, Peradenia; Voyage A. Humbert.

$=$ Dryophis pulverulentus pulverulentus $\mathrm{D} . \& \mathrm{~B}$.

Echis carinatus leakeyi Stemmler et Sochurek

1969 Aquaterra 6 (8): 89-93, 2 fig.

Paratypes: MHNG 1324.26-30.

4 ô, 1 f , Kenya, lac Baringo, bord ouest, (coll. Kramer 10014, 12136, 12137, 12138, 12139).

Echis carinatus sochureki Stemmler

1969 Aquaterra 6 (10): 118-124, Abb. 1-4.

Paratypes: MHNG 1324.31-36.

4 ㅇ, 2 ^̊, Iran, Zabel; M. Latifi, 1965 (coll. Kramer 9070, 0614, 0618, 0073, 0074, 0084).

Paratype: MHNG 1324.38.

1 ô, West-Pakistan (Kramer 10091).

Paratypes: MHNG 1324.39-40.

2 ex., URSS, Turkmenistan, Imam-Baba, 1962 (Kramer 1880, 1881).

Paratypes: MHNG 1324.41-43.

1 §ิ, 2 +, URSS, Turkmenistan, Marotskaja Prov., 1962 (Kramer 2769, 2770, 3314).

\section{Elaphis dione temporalis Jan}

1863 Elenc. sist. Ofidi : 61.

Holotype: MHNG 16.53.

1 ex., Origine ?; M. Moricand.

\section{Elapotinus picteti Jan}

1862 Arch. Zool. Anat. Fisiol. Genova 2 (1): 31-32.

Holotype: MHNG 279.37.

1 ex., Origine: Guyane ?; M. Pelletier, X.1840. 
Dans le même bocal et sous le même numéro se trouve encore un deuxième exemplaire, non mentionné dans la publication originale.

\section{Enicognathus humberti Jan}

1863 Arch. Zool. Anat. Fisiol. Modena 2 (2): 275-276.

Holotype: MHNG 523.92.

1 ô, Ceylan, Trincomalie; Voyage A. Humbert, 1861.

= Sibynophis subpunctatus $($ D. \& B.)

\section{Enicognathus occipitalis Jan}

1863 Arch. Zool. Anat. Fisiol. Modena 2 (2): 267.

Syntype: MHNG 179.42.

1 ex., Brésil, Bahia; M. F. Favre-Bertrand, VI.1835.

$=$ Liophis occipitalis $(\mathrm{Jan})$

\section{Erythrolampus aesculapii bizona Jan}

1863 Arch. Zool. Anat. Fisiol. Modena 2 (2): 315.

Syntype: MHNG 464.30.

1 ex., Origine: Bahia ?, Brésil.

\section{Erythrolampus aesculapii dicranta Jan}

1863 Arch. Zool. Anat. Fisiol. Modena 2 (2): 315.

Syntype: MHNG 524.49.

1 ex., Brésil, Bahia; M. Blanchet, 1861 ?

\section{Glauconia hamulirostris Nikolski}

1907 Annuaire Mus. Zool. Acad. impér. Sci. St. Pétersbourg 10 (1905): 286-288, fig. 4,5 .

Syntype: MHNG 1326.72.

1 ex., Iran, Aguljaschker, 49 40' ö.L., 31 $40^{\prime}$ n.B.; A. N. Zaroudny, 28.III.1904 (Mus. Leningrade $\mathrm{n}^{\circ} .10299$ - coll. Kramer $\mathrm{n}^{\circ}$. 3986).

$=$ Leptotyphlops hamulirostris (Nikolski)

\section{Helicops pastazae Shreve}

1934 Occ. Pap. Boston Soc. Nat. Hist. 8: 129.

Paratype: MHNG 1069.97.

1 ex., Equateur, Pastaza Riv., entre Canelos et Maranon River.

\section{Homalosoma melanocephalum Jan}

1862 Arch. Zool. Anat. Fisiol. Genova 2 (1): 34.

Syntype: MHNG 1246.77.

1 ex., Liban, Beyrouth; M. Sordet.

$=$ Rhynchocalamus melanocephalus $(\mathrm{Jan})$ 


\section{Liophis reginae ornata Jan}

1863 Arch. Zool. Anat. Fisiol. Modena 2 (2): 295-296.

Holotype: MHNG 180.39.

1 ex., Argentine, Buenos Aires; M. Bacle, 1835.

$=$ Leimadophis reginae ornata $(\mathrm{Jan})$

\section{Liparophis bedoti Peracca}

1904 Revue suisse Zool. 12: 663-665.

Holotype: MHNG 677.45.

1 , Chine, Tung-kun, Canton; Kühne, 1903 (?).

= Opisthotropis balteatus (Cope)

\section{Micrurus mertensi Schmidt}

1936 Zool. Ser. Field Mus. Nat. Hist. 20, no 19: 192-193.

Paratype: MHNG 1327.93.

1 ex., Perou, Pacasmayo; M. Boulenger, 1887 (coll. Kramer 10127, éch. Mus. Senckenberg $n^{\circ}$ 20713).

\section{Naja tripudians paucisquamis Peracca}

1899 Revue suisse Zool. 7: 328.

Holotype: MHNG 748.43.

1 ex., Borneo, Sarawak; M. Bedot-C. Pictet.

= Naja naja paucisquamis Peracca

\section{Natrix natrix lanzai Kramer}

1970 Lavori Soc. ital. Biogeografia, N.S. 1 : 671-673, Taf. II, fig. 6, Taf. IV, fig. 9-12. Holotype: MHNG 1324.94.

1 ô, Italie, Dintorni delle "Croci di Calenzano », Comune di Calenzano, prov. de Florence, entro le acque del torrente Ritortolo, affluente di destra del fiume Sieve, 290 m.; B. Lanza, 11.VII.1970 (coll. Kramer 12422).

Paratypes: MHNG 1324.95-100, 1325.1-23, 1382.89-99, 1396.9.

17 ô, 24 +, (localités: env. Rome: Ostia antica, lac Albano; env. Florence; Siena; env. Grosseto; Porto Santo Stefano; Battipaglia Salerno; Lago di Patria/ Naples; Rimini; Monte Argentario); B. Lanza, J. Kretz, E. Kramer, E. Granchi, G. B. Toscanelli, 1960-1970 (coll. Kramer 2423, 2675, 2676, 3564, 3700, 3702, $3708,3709,3710,3711,3712,3781,3793,3794,3809,3811,3813,3819,3823$, 4046, 4047, 4048, 4049, 5076, 5079, 5085, 5086, 5088, 5089, 5656, 5657, 5658, $5693,11271,11272,12148)$.

\section{Oligodon ornatus Roux}

1914 Revue suisse Zool. 22: 28.

Holotype: MHNG 767.2.

1 ex., Sumatra.

= Oligodon petronellae Roux 


\section{Opisthotropis kuatunensis Pope}

1928 Amer. Mus. Novit., no. 320: 5.

Paratype: MHNG 1369.46.

1 ex., Chine, Chungan Hsien, Fukien, 3rd Asiatic Expedition; C. H. Pope, IV.IX.1926 (Am. Mus. Nat. Hist. 34433).

\section{Oxyrhopus bicolor Peracca}

1904 Revue suisse Zool. 12: 667-668.

Holotype: MHNG 677.47.

1 ô, Argentine, nord Santa Fé, Buenos-Aires.

$=$ Clelia bicolor (Peracca)

\section{Psammophis elegans univittatus Perret}

1961 Bull. Soc. Neuchâtel. Sci. nat. 84: 136.

Holotype: MHNG 1213.92.

1 , Cameroun, Zidim, $50 \mathrm{~km} \mathrm{~W}$ de Maroua, 28.XII.1960. J.-L. Perret.

Paratype: MHNG 1213.93.

1 q, même provenance, 29.XII.1960.

Paratype: MHNG 1213.94.

1 \&, même provenance, 31.XII.1960.

Paratype: MHNG 1213.95.

1 ㅇ, même provenance, 4.I.1961.

Paratype: MHNG 1213.96.

1 \&, même provenance, $30 .$. XII.1960.

\section{Rabdosoma trivirgatum Jan}

1862 Arch. Zool. Anat. Fisiol. Genova, 2 (1): 17.

Syntype: MHNG 524.2.

1 ex., Origine: Amérique du Sud ?

$=$ Atractus trilienatus Wagler

\section{Trachischium laeve Peracca}

1904 Revue suisse Zool. 12: 665-666.

Holotype: MHNG 677.46.

1 , Indes orientales; H. de Saussure.

Le mâle noté dans la description reste introuvable.

Tropidonotus natrix picturata Jan

1864 Arch. Zool. Anat. Fisiol. Modena 3: 204, 209.

Syntype: MHNG 523.97.

1 ex., Origine?

$=$ Natrix natrix scutata (Pallas) 


\section{Vipera aspis zinnikeri Kramer}

1958 Vierteljahrschr. naturf. Ges. Zürich 103: 323, Abb. 1, 2.

Paratypes: MHNG 1324.14-25.

6 ô, 6 q , France, Auch, Dép. Gers, (Garonne-Becken) W. Zinniker, Sommer 19571958. (coll. Kramer no. 203, 205, 206, 210, 214, 216, 328, 372, 397, 443, 445, 446).

\section{Vipera latifi Mertens, Darevsky et Klemmer}

1967 Senckenberg. biol. 48 (3): 161-168, fig. 5.

Paratypes: MHNG 1324.44-45.

2 을 Iran, nordöstlich von Teheran, Hochtal von Lar, $2180-2900 \mathrm{~m}$; südwestlich des Demavend-Gipfels im Elburs-Gebirge; M. Latifi (coll. Kramer 9071, 9072).

\section{Vipera latastei monticola Saint-Girons}

1953 Bull. Soc. zool. Fr. 78: 24-25.

Syntype: MHNG 1353.7.

1 ô, Maroc, Haute Atlas, Massif du Toubka, vallée de l'oued Réraia, 3100 m., flanc Sud-Est d'une épaule de l'Aguelzim; H. Saint-Girons, 1952 (coll. Kramer 1239).

\section{BIBLIOGRAPHIE}

\section{POISSONS}

ABE, T. 1961. Notes on some fishes of the subfamily Braminae, with the introduction of a new genus, Pseudotaractes. Jap. J. Ichthyol. 8: 92-99.

Chabanaud, P. 1930. Les Genres de Poissons Hétérosomates (Pisces Heterosomata) appartenant à la sous-famille des Soleinae. Bull. Inst. océan. Monaco 555: 1-23.

FANG, P. W. 1936. Study on the Botoid Fishes of China. Sinensia 7 (1): 1-49.

Hubbs, C. L. 1936. Fishes on the Yucatan Peninsula. Publ. Carnegie Inst. Washington 457: 157-287.

JAYARAM, K. C. 1954. Siluroid fishes of India, Burma and Ceylon. XIV. Fishes of the genus Mystus Scopoli. Rec. Indian Mus. 51: 527-558.

JaYARAM, K. C. 1974. Contribution to the study of Bagrid fishes. XI. Designation of a Lectotype for Macrones argentivittatus with notes on some Bagrid species in the collections of the Natural History Museum, Genève. Revue suisse Zool. 81 (4): 785-790.

Pellegrin, J. 1914. Sur une dernière collection de poisson recueillie à Madagascar par feu F. Geay. Bull. Mus. natn. Hist. nat. 20: 111-113.

Reichel, M. 1927. Etude anatomique du Phreatobius cisternarum Goeldi, Silure aveugle du Brésil. Revue suisse Zool. 34: 285-403, 4 pl.

RobBins, C. R. 1964. Sciaena bedoti and Corvina subaequalis junior synonyms of Bairdiella rochus, a western Atlantic Sciaenid fish. Annls. Mag. nat. Hist., ser. 13, 7: 705-706.

Schultz, L. P., E. S. Herald, E. A. Lachner, A. D. Welander and L. P. Woods. 1953. Fishes of the Marshall Marianas Islands. I. Bull. U.S. nat. Mus. 202: xxxii +385 pp.

SмiтH, J. L. B. 1957. List of Fishes of the family Labridae in the Western Indian Ocean with new records and five new species. Ichthyol. Bull. Rhodes Univ. 7: 99-114.

Sмiтн, J. L. B. 1962. The moray eels of the Western Indian Ocean and the Red Sea. Ichthyol. Bull. Rhodes Univ. 23: 421-444.

Stehmann, M. 1974. Pteroplatea binotata Lunel, 1879, ein vergessenes Juniorsynonym von Gymnura altavela (Linnaeus, 1758) (Pisces, Batoidei, Gymnuridae). Revue suisse Zool. 81 (1): 83-93. 


\section{AMPHIBIENS}

Aмiet, J.-L. 1971. Espèces nouvelles ou mal connues de Leptodactylodon (Amphibiens Anoures) de la Dorsale camerounaise. Annls. Fac. Sci. Cameroun 5: 57-81.

AmiEt, J.-L. 1973. Notes faunistiques, éthologiques et écologiques sur quelques Amphibiens Anoures du Cameroun (2e série). Annls. Fac. Sci. Cameroun 13: 135-161.

AmiEt, J.-L.1976. Observations anatomiques et biologiques sur le genre Werneria Poche, 1903 (Amphibiens Anoures, fam. Bufonidae). Revue Zool. Bot. afr. 90 (1): 33-45.

Amiet, J.-L. et A. Schiøtz. 1974. Voix d'Amphibiens camerounais III. Hyperoliinae: genre Leptopelis. Annls. Fac. Sci. Cameroun 17: 131-163.

GuibÉ, J. et M. Lamotte. 1957. Révision systématique des Ptychadena (Batraciens Anoures Ranidés) d'Afrique occidentale. Bull. IFAN, 29, sér. A: 937-1003.

GuibÉ, J. et M. LAmotte. 1963. La Réserve naturelle intégrale du Mont Nimba. XXVIII. Batraciens du genre Phrynobatrachus. Mém. IFAN 66: 601-627.

Lamotte, X. et F. Xavier. 1966. Etude comparée de deux espèces de Phrynobatrachus souvent confondues: Phr. plicatus Günther et Phr. auritus Boulenger. Bull. IFAN 28, sér. A: 1605-1619.

Laurent, R. F. 1951a. Catalogue des rainettes africaines (genres Afrixalus et Hyperolius) de la collection du Museum national d'Histoire naturelle de Paris. Annls. Soc. $r$. zool. Belgique 82: 23-50.

LaURent, R. F. 1951 b. Aperçu des formes actuellement reconnaissables dans la superespèce Hyperolius marmoratus. Annls. Soc. r. zool. Belgique 82 (2): 379-397.

Laurent, R. F. 1954. Remarques sur le genre Schoutedenella Witte. Annls. Mus. roy. Congo belge, N.S. 4, Zool. 1: 34-40.

LaURent, R. F. 1958. La réserve intégrale du Mont Nimba XIII. Les Rainettes du genre Hyperolius. Mém. IFAN 53: 275-299.

Perret, J.-L. 1966. Les Amphibiens du Cameroun. Zool. Jb. Syst. 93: 289-464.

PERRET, J.-L. 1972. Les espèces des genres Wolterstorffina et Nectophrynoides d'Afrique (Amphibia Bufonidae). Annls. Fac. Sci. Cameroun 11: 93-119.

Perret, J.-L. 1973. Leptopelis palmatus (Peters) et Leptopelis rufus (Reichenow) (Amphibia Salientia): deux espèces distinctes. Annls. Fac. Sci. Cameroun 15-16: 81-90.

Perret, J.-L. 1975. Revision critique de quelques types de reptiles et batraciens africains. Revue suisse Zool. 82 (1): 185-192.

Perret, J.-L. 1976. Identité de quelques Afrixalus (Amphibia, Salientia, Hyperoliidae). Bull. Soc. Neuchatel. Sci. nat.

Schiøтz, A. 1971. The Superspecies Hyperolius viridiflavus (Anura). Vidensk. Medd. Dansk. Naturh. Foren. 134: 21-76.

\section{REPTILES}

FuHN, I. E. 1970. Contribution à la systématique des Lygosominés africains (Reptılia, Scincidae).

I. Les espèces attribuées au genre Ablepharus. Revue roum. Biol.-Zool. 15 (6): 379-393.

Funn, I. E. 1972. Révision du Phylum forestier du genre Panaspis Cope (Reptilia, Scincidae, Lygosominae). Revue roum. Biol.-Zool. 17 (4): 257-271.

Greer, A. E. Jr. 1974. The Generic Relationships of the Scincid Lizard Genus Leiolopisma and its Relatives. Austral. J. Zool., Suppl. Ser. 31: 1-67.

INGER, R. F. and H. MARX. 1965. The systematics and evolution of the oriental colubrid snakes of the genus Calamaria. Fieldiana Zool. 49: 304 pp.

Papenfuss, Тн. J. 1969. Preliminary Analysis of the Reptiles of arid Central West Africa. Wasman J. Biol. 27 (2): 249-325.

Pasteur, G. 1964. Recherches sur l'évolution des Lygodactyles, lézards afro-malgaches actuels. Trav. Inst. Sci. Chérif. Rabat, sér. Zool. 29: 132 pp. 
Peters, J. A. and B. Orejas-Miranda. 1970. Catalogue of the Neotropical Squamata. Part I. Snakes. U.S. Nat. Mus. Bull. 297: viii + 347 pp.

Pope, C. 1935. The Reptiles of China. Nat. Hist. Central Asia, vol. 10: 604 pp.

RooIJ, N. DE. 1917. The Reptiles of the Indo-Australian Archipelago, II. Ophidia. E. J. Brill, Leiden: $x i v+334 \mathrm{pp}$.

Schmidt, K. P. 1939. Reptiles and Amphibians from Southwestern Asia. Field Mus. Publ. Chicago, Zool. 24: 49-92.

Sмiтн, M. A. 1943. Reptilia and Amphibia.-Serpents. Fauna of British India, vol. 3: xii +583 pp. TAYlor, E. H. 1950. The Snakes of Ceylon. Univ. Kansas Science Bull. 33 (14): 519-603.

Thys van Den Audenaerde, D. F. E. 1967. Les Gekkonidae de l'Afrique centrale. Revue Zool. Bot. afr. 76 (1-2): 163-172.

Uzzel, Th. M. Jr. 1966. Teiid Lizards of the Genus Neusticurus (Reptilia, Sauria). Bull. Am. Mus. nat. Hist. 132 (5): 277-328.

Adresse de l'auteur :

Muséum d'Histoire naturelle

case postale 284

CH-1211 Genève 6

Suisse 


\section{$2 \mathrm{BHL}$ Biodiversity Heritage Library}

Mahnert, Volker. 1976. "Catalogue des types de poissons, amphibiens et reptiles du Muséum d'Histoire naturelle de Genève." Revue suisse de zoologie 83, 471-496. https://doi.org/10.5962/bhl.part.118840.

View This Item Online: https://www.biodiversitylibrary.org/item/129622

DOI: https://doi.org/10.5962/bhl.part.118840

Permalink: https://www.biodiversitylibrary.org/partpdf/118840

\section{Holding Institution}

Smithsonian Libraries

\section{Sponsored by}

Biodiversity Heritage Library

\section{Copyright \& Reuse}

Copyright Status: In Copyright. Digitized with the permission of the rights holder.

Rights Holder: Muséum d'histoire naturelle - Ville de Genève License: http://creativecommons.org/licenses/by-nc-sa/3.0/

Rights: https://www.biodiversitylibrary.org/permissions/

This document was created from content at the Biodiversity Heritage Library, the world's largest open access digital library for biodiversity literature and archives. Visit BHL at https://www.biodiversitylibrary.org. 